Soraya Benamar ${ }^{1}$, Charlotte $\mathrm{Hua}^{2}$, Jacques Morel ${ }^{3}$, Francoise Flaisler ${ }^{4}$, Bernard Combe ${ }^{1}$, Cédric Lukas ${ }^{1}$, Cecile Gaujoux-Viala ${ }^{5} .{ }^{1}$ Montpellier University, Rheumatology, Montpellier, France; ${ }^{2}$ Montpellier University, Nimes, France; ${ }^{3}$ Montpellier University, Rheumatology, Montpellier, France; ${ }^{4}$ Montpellier University, Nimes, France; ${ }^{5}$ Montpellier University EA 2415, Nimes, France

Background: Rheumatic disorders (RD) provide chronic pain. Catastrophizing is a negative cognitive and affective response resulting in inadequate expression of pain. Catastrophizing in RD patients is suspected to be involved in persistence and amplification of chronic pain.

Objectives: To assess catastrophizing level in RD and to evaluate the association between catastrophizing and physical pain intensity, disease activity, disability, depression and quality of life.

Methods: We performed a systematic review of literature and search in the following databases: MEDLINE, COCHRANE and EMBASE until April 2018. All observational, cross-sectional and randomized control studies investigating catastrophizing in patients with RD (rheumatoid arthritis (RA), low back pain (LBP), osteoarthritis) were included. Statistical analysis defined pooled mean catastrophizing level by using the Pain Catastrophizing Scale (PCS), the latter ranging from 0 to 52. To assess the association between catastrophizing and disease activity (DAS28), pain (Numerical Rating Scale NRS), disability (ODI) and quality of life (SF36, WOMAC) we collected correlation coefficients and pooled them in metaanalysis using the Fisher's $\mathrm{z}$ transformation with MedCalc v18.11.3.

Results: From 1494 articles concerning catastrophizing and RD, 51 were selected for a meta-analysis :

- 601 RA patients (mean age 57.4 years, $67.7 \%$ female, mean pooled DAS $28=3.4$ and mean pooled NRS $=3.8$ ) included in 7 studies. The mean pooled catastrophizing level at baseline was $14.7(\mathrm{SD}=11.4)$ in RA patients vs $2.7(S D=3.0)$ in control group patients $(n=82$ in 2 studies). In one study, a RA sample identified $22 \%$ of high catastrophizers (defined by PCS > or $=30$ ).

- 2521 LBP patients (mean age 43.6 years, 57\% female, mean pooled NRS $=4.5$ and mean pooled ODI $=30.3$ ) included in 27 studies. The mean pooled catastrophizing level at baseline was $19.9(\mathrm{SD}=11.3)$.

- 3388 osteoarthritis patients (knee and hip, mean age 67.7 years, 65\% female, mean pooled NRS $=5.0$ ) included in 17 studies. The mean pooled catastrophizing level at baseline was $11.2(S D=10)$.

In RA, a significant positive correlation between catastrophizing and DAS 28 was observed: pooled $r=0.278 p<0.001$ ( 3 studies) (Figure 1). Pain level was strongly associated with catastrophizing $(r=0.71 \quad(p<0.01)$. Higher PCS scores were significantly associated with higher levels of distress $i$. e. lower SF-36 Mental Health score $(r=-0.52 \quad(p<0.01))$ and significantly associated with reduced physical function $(r=-0.35 \quad(p<0.01)$ for SF36 Physical Function).

In LBP, higher PCS scores were significantly associated with higher levels of pain and disability : pooled $r=0.486 \quad p<0.001$ (NRS) and $r=0.465$ $\mathrm{p}<0.001$ (ODI), respectively. The association between catastrophizing and depression was significant $(r=0.538 \quad p<0.01)$.

Catastrophizing in osteoarthritis was strongly associated with an increase in functional limitations and pain (i.e. higher WOMAC total score, correlation coefficient: $r=0.641 \quad p<0.001$ ).

Conclusion: Catastrophizing is a common psychological trait clearly associated with disease activity, pain, mental health and physical function. Nevertheless catastrophizing is rarely measured. It might be relevant to detect it earlier in order to adapt pharmacologic and non-pharmacologic treatment in at-risk patients.

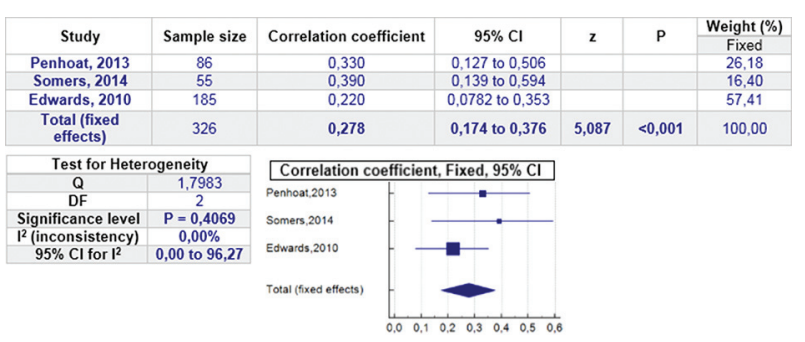

Figure 1. Forest plot for correlation coefficient between catastrophizing (PCS) and disease activity (DAS 28) in rheumatoid arthritis

Disclosure of Interests: Soraya Benamar: None declared, Charlotte Hua: None declared, Jacques Morel: None declared, Francoise Flaisler: None declared, Bernard Combe Consultant for: Abbvie, Bristol-Myers Squibb, Gilead, Janssen, Eli Lilly, MSD, Novartis, Pfizer, Roche-Chugai, Sanofi,
UCB, Cédric Lukas: None declared, Cecile Gaujoux-Viala Consultant for: Speaking and/or consulting fees from AbbVie, Amgen, Bristol-Myers Squibb, Celgene, Eli Lilly, Gilead, Janssen, Merck-Serono, Medac, Nordic Pharma, Novartis, Pfizer, Roche, Sandoz, Sanofi and UCB Pharma., Speakers bureau: Speaking and/or consulting fees from AbbVie, Amgen, Bristol-Myers Squibb, Celgene, Eli Lilly, Gilead, Janssen, Merck-Serono, Medac, Nordic Pharma, Novartis, Pfizer, Roche, Sandoz, Sanofi and UCB Pharma.

DOI: 10.1136/annrheumdis-2019-eular.5891

\section{THU0660 CHARACTERIZATION OF SCLERODERMA PATIENTS ACCORDING TO RO52 AND KU ANTIBODIES}

Julien Caballero Castro ${ }^{1}$, Alfredo Guillén del Castillo ${ }^{2}$, L Eduardo. CallejasMoraga $^{3}$, Dolores Colunga Argüelles ${ }^{4}$, Luis Sáez-Comet ${ }^{5}$, Manuel Rubio-Rivas ${ }^{6}$, Ana Argibay ${ }^{7}$, Jose Antonio Vargas-Hitos ${ }^{8}$, Jose Antonio Todolí Parra ${ }^{9}$, Isabel Perales Fraile ${ }^{10}$, Carmen Pilar Simeón-Aznar ${ }^{2}$, On Behalf of Rescle Investigators, Autoimmune Diseases Study Group (Geas) ${ }^{11} .{ }^{1}$ Hospital Obispo Polanco, Department of Internal Medicine, Teruel, Spain; ${ }^{2}$ Hospital Universitario Vall d'Hebron, Unit of Autoimmune Diseases, Department of Internal Medicine, Barcelona, Spain; ${ }^{3}$ Corporación Sanitaria Universitaria Parc Taulí, Department of Internal Medicine, Barcelona, Spain; ${ }^{4}$ Hospital Universitario Central de Asturias, Department of Internal Medicine, Oviedo, Spain; ${ }^{5}$ Hospital Universitario Miguel Servet, Department of Internal Medicine, Zaragoza, Spain; ${ }^{6}$ Hospital Universitario de Bellvitge-IDIBELL, Unit of Autoimmune Diseases, Department of Internal Medicine, Barcelona, Spain; ${ }^{7}$ Complejo Hospitalario Universitario de Vigo, Unit of Systemic Autoimmune Diseases and Thrombosis. Department of Internal Medicine, Vigo, Spain; ${ }^{8}$ Hospital Universitario Virgen de las Nieves, Department of Internal Medicine, Granada, Spain; ${ }^{9}$ Hospital Universitario y Politécnico La Fe, Department of Internal Medicine, Valencia, Spain; ${ }^{10}$ Hospital Universitario Rey Juan Carlos. Móstoles, Department of Internal Medicine, MADRID, Spain;

${ }^{11}$ Sociedad Española de Medicina Interna, Madrid, Spain

Background: Systemic Sclerosis (SSc) is an autoimmune desease that is characterized by progressive and severe fibrosis with cutaneous and visceral involvement, fibroproliferative vasculopathy and alterations of cellular and humoral immunity, very heterogenous from the clinical and immunological point of view. There are many types of antibodies related to the disease that are used in the diagnosis and characterization. Antibodies anti-Ku and anti-Ro52 have been found, although they are not specific to the disease, that could play an important role in the prognosis and clinical expression.

Objectives: To evaluate the clinical pattern and prognosis of SSc patients who carry antibodies Anti-Ku and Anti-Ro52.

Methods: A retrospective, multicentric study of SSc patients included in the Spanish Registry of Scleroderma (RESCLE). Clinical, demographic, prevalence, serological, and survival data were analyzed

Results: A total of 401 samples were analyzed for anti-Ku with 12 positive results $(3 \%)$. For anti-R052, 1724 sampled were analyzed with 246 positive results $(14 \%)$. It is observed, from the multivariant analysis, that patients with anti-Ro52 presented, in higher frequency, an association with Sicca syndrome $(p<0.001)$ and its coexistence with the antibody antiLa (OR 44.8 with $95 \% \mathrm{Cl}$ and $\mathrm{p}<0.001$ ). Regarding patients with anti-Ku, no clinical association was found with statistical significance.

Conclusion: As opposed to finding SSc specific antinbodies such as anticentromere (ACA), anti-topoisomerase I (ATA), and anti-RNA polymerase III (ARA), the presence of anti-Ku or anti-Ro52 is not conclusive of any distinctive clinical profile.

\section{REFERENCES:}

[1] Mehra S., et al. Autoantibodies in systemic sclerosis. Autoinmunity Reviews 2013; 12:340-354.

[2] Stern E., Denton C. The Pathogenesis of systemic Sclerosis. Rheum Dis Clin North Am 2015; 41(3):367-82.

[3] Cavazzana I., et al. A subset of systemic sclerosis but not of systemic lupus erythematosus is defined by isolated anti-Ku autoantibodies. Clin Exp Rheumatology 2013; 31:118-21.

[4] Iniesta N.; et al. Influence of antibody profile in clinical features and prognosis in a cohort of Spanish patients with systemic sclerosis. Clin Exp Rheumatology 2017; 35(suppl. 106)S00-S00.

Disclosure of Interests: None declared DOI: 10.1136/annrheumdis-2019-eular.8098 